\title{
Aggregation-Robustness and Model Uncertainty of Regulatory Risk Measures
}

\author{
Paul Embrechts,; Bin Wang ${ }^{\dagger}$ and Ruodu Wang ${ }^{\ddagger}$
}

February 10, 2014

\begin{abstract}
Research related to aggregation, robustness, and model uncertainty of regulatory risk measures, for instance, Value-at-Risk (VaR) and Expected Shortfall (ES), is of fundamental importance within quantitative risk management. In risk aggregation, marginal risks and their dependence structure are often modeled separately, leading to uncertainty arising at the level of a joint model. In this paper, we introduce a notion of qualitative robustness for risk measures, concerning the sensitivity of a risk measure to the uncertainty of dependence in risk aggregation. It turns out that coherent risk measures, such as ES, are more robust than VaR according to the new notion of robustness. We also give approximations and inequalities for aggregation and diversification of VaR under dependence uncertainty, and derive an asymptotic equivalence for worst-case VaR and ES under general conditions. We obtain that for a portfolio of a large number of risks VaR generally has a larger uncertainty spread compared to ES. The results warn that unjustified diversification arguments for VaR used in risk management need to be taken with much care, and potentially support the use of ES in risk aggregation. This in particular reflects on the discussions in the recent consultative documents by the Basel Committee on Banking Supervision.
\end{abstract}

Key-words: Value-at-Risk; Expected Shortfall; dependence uncertainty; risk aggregation; robustness; inhomogeneous portfolio; Basel III.

\section{Introduction}

Risk measurement, with its crucial importance for financial institutions such as banks, insurance companies and investment funds, has drawn a lot of attention in both academia and

\footnotetext{
*RiskLab and SFI, Department of Mathematics, ETH Zurich, 8092 Zurich, Switzerland.

${ }^{\dagger}$ Department of Mathematics, Beijing Technology and Business University, Beijing 100048, China.

${ }^{\ddagger}$ Corresponding author. Department of Statistics and Actuarial Science, University of Waterloo, ON N2L3G1, Canada. Email: wang@uwaterloo.ca
} 
industry over the past several decades. Although a financial risk, often modeled by a probability distribution, cannot be characterized by a single number, sometimes one needs. The determination of regulatory capital is one such example, the ranking of risks another. For such purposes, quantitative tools that map risks to numbers were introduced, and they are called risk measures.

Over the past three decades, Value-at-Risk (VaR) became the benchmark (Jorion (2006)). Expected Shortfall (ES), an alternative to VaR which is coherent, (Artzner et al. (1999)), is arguably the second most popular risk measure in use. In two recent consultative documents BCBS (2012, 2013), the Basel Committee on Banking Supervision proposed to take a move from VaR to ES for risk measurement in banking. Under Solvency 2 and the Swiss Solvency Test, the same discussion takes place within insurance regulation; see for instance Sandström (2010) and SCOR (2008). As a consequence, there have been extensive debates on issues related to diversification, aggregation, economical interpretation, extreme behavior, robustness, and backtesting of VaR and ES. We omit a detailed analysis here and refer to Embrechts et al. (2014), Emmer et al. (2014) and the reference therein.

Here are some of the issues raised: VaR is not coherent, but it is elicitable (Gneiting (2011)), easy to backtest and more robust with respect to statistical uncertainty, as argued in Gneiting (2011) and Cont et al. (2010); ES is coherent, but not elicitable, difficult to backtest and less robust. There have been extensive discussions on the problematic diversification and aggregation issues of VaR due to its lack of subadditivitity; see for example Embrechts et al. (2013). Daníelsson et al. (2005) argue that the violation of subadditivity for VaR is rare in practice. VaR, being a quantile, does not address the crucial "what if" question. Whereas this was clear since its introduction within the financial industry in 1994, it took some serious financial crises to bring this issue fully onto the regulatory agenda.

The importance of robustness properties of risk measures has only fairly recently become a focal point of regulatory attention. By now, numerous academic as well as applied papers address the topic. Conflicting views typically result from different notions of robustness; Embrechts et al. (2014) contains a brief discussion and some references. In this paper, issues related to both aggregation and robustness for VaR and ES will be discussed. We will show that ES indeed enjoys a new notion of robustness which VaR generally does not.

The mathematical property of (non-)subaddivity of a risk measure becomes relevant upon analyzing the aggregate position of a portfolio. As often is the case in practice, the dependence structure among individual risks in a portfolio is difficult to obtain from a statistical point of view, while the marginal distributions of the individual risks (assets) may typically be easier to model; see for instance Embrechts et al. (2013) and Bernard et al. (2014). Modeling a high-dimensional dependence structure is well-known to be data-costly, and dimension reduction 
techniques such as vine copulas, hierarchical structures, and very specific parametric models often have to be implemented. Whereas such simplifying techniques in general create computational and modeling ease, they typically involve considerable model uncertainty. This leads to a notion of dependence uncertainty (DU) in risk aggregation, a concept of main interest for this paper.

From a mathematical or statistical point of view it is clearly better to look at the robustness properties of a model at the level of the joint distribution of the risk factors. The main reason for separating the two (marginals, dependence) is because of processes in practice, where indeed the two are often modeled separately. This is particularly true in a stress testing environment.

Hence for this paper, we introduce the notion of aggregation-robustness to study the robustness properties of risk measures for aggregation in the presence of dependence uncertainty. The new notion of robustness is consistent with the classic notion of robustness for statistical functionals in e.g. Huber and Ronchetti (2009), and is weaker than the robustness for risk measures for instance defined in Cont et al. (2010). However, as opposed to the conclusions in the latter paper, we show that when the model uncertainty lies in the dependence structure, coherent distortion risk measures (such as ES) are robust, whereas VaR in general is not. This result supports the use of ES for risk aggregation, especially when statistical information on marginal distributions is reliable.

Under DU, the values of VaR and ES lie in an interval. This interval can be seen as a measurement of model uncertainty for a particular risk measure. When a risk measure is applied to an aggregate position of a portfolio, the ratio between the risk measure of the aggregate risk and the summation of the risk measures of the marginal risks, is called a diversification ratio. The diversification ratio measures how good the risks in a portfolio hedge (compensate for) each other. With only models for marginal distributions available, the diversification ratio also lies in a DU-interval.

To study the DU-interval of VaR and ES, and their diversification ratios, one needs to calculate the worst-case and best-case values of VaR and ES under dependence uncertainty. Due to the subadditivity of ES, the worst-case value of ES is the summation of the ES of the marginal risks. However, the other three quantities (best- and worst-case VaR, best-case ES) are, in general, unknown. Partial results do exist. The worst-case values of VaR for $n=2$ were given in Makarov (1981) based on early results in multivariate probability theory. Embrechts and Puccetti (2006) gave a dual bound for the worst-case VaR for $n \geqslant 3$ in the homogeneous model, i.e. all marginal risks have the same distribution. Partial solutions for the worst-case and best-case values of VaR are to be found in Wang et al. (2013), Puccetti and Rüschendorf (2013) and Bernard et al. (2014), based on the notion of complete mixability introduced in Wang and Wang (2011). A fast algorithm to numerically calculate the worst-case and best-case values of 
VaR under general conditions was introduced in Embrechts et al. (2013). For the best-case ES, some partial analytical results can be found in Bernard et al. (2014) and Cheung and Lo (2013), and a numerical procedure was proposed by Puccetti (2013).

In most of the existing analytical results, it is assumed that the marginal distributions have to be identical (homogeneous case), with some extra conditions on the shape of the underlying risk factor densities (assumed to exist). In this paper, we relax the assumptions on the marginal distributions. Instead of explicit values for the worst-case and best-case VaR, we obtain approximations. The new results obtained can be used within a discussion on capital requirement; they moreover yield a DU-interval for VaR and its diversification ratio.

Further understanding of the worst-case VaR can be obtained through the asymptotic behavior as the number of risks in the portfolio grows to infinity, i.e. a large portfolio regime. In the homogeneous case, Puccetti and Rüschendorf (2014) obtained an asymptotic equivalence between the worst-case VaR and the worst-case ES under dependence uncertainty, and this under a strong condition of complete mixability. The condition on the identical marginal distributions was later weakened by Puccetti et al. (2013) (based on further results on complete mixability) and Wang (2014) (based on a duality theory in Rüschendorf (1982)). It was finally removed by Wang and Wang (2014) (based on the new notion of extremely negative dependence). When the marginal distributions are not identical, Puccetti et al. (2013) also obtained the asymptotic equivalence under the assumption that only finitely many different choices of the marginal distributions can appear; this mathematically allows a reduction to the case of identical marginal distributions.

In this paper, we give a unifying result on this asymptotic equivalence, by allowing the marginal distributions to be arbitrary. Only weak uniformity conditions on the moments of the marginal distributions are required for our result to hold. These conditions are easily justified in practice, and are necessary for the most general equivalence to hold. The new results lead to the asymptotic DU-spread of VaR and ES, and show that VaR in general yields a larger DU-spread compared to ES.

We hope that the results in this paper would successfully deliver the following message:

With respect to dependence uncertainty in aggregation, VaR is less robust compared to ES.

The rest of the paper is organized as follows. In Section 2 we introduce the notion of aggregation-robustness and show that ES is aggregation-robust but VaR is not. In Section 3 we give new bounds on the diversification ratios under dependence uncertainty, and establish an asymptotic equivalence between VaR and ES under a worst-case scenario. The dependence uncertainty spread of VaR and that of ES are derived and compared in Section 4. In Section 
5, numerical examples are presented to illustrate our results. Section 6 draws some conclusions. All proofs are put in the Appendix.

Throughout the paper, we let $(\Omega, \mathcal{A}, \mathbb{P})$ be a standard atomless probability space and $L^{0}:=L^{0}(\Omega, \mathcal{A}, \mathbb{P})$ be the set of all real-valued random variables (rvs) on that probability space. Elements of $L^{0}$, rvs, will often be referred to as risks. Their distribution functions we simply refer to as distributions. We write $X \sim F$ to denote $F(x)=\mathbb{P}(X \leqslant x), x \in \mathbb{R}$. We also denote the generalized inverse function of $F$ by $F^{-1}(p)$, that is $F^{-1}(p)=\inf \{t \in \mathbb{R}: F(t) \geqslant p\}$ for $p \in(0,1]$, and $F^{-1}(0)=\inf \{t \in \mathbb{R}: F(t)>0\}$.

\section{Robustness of VaR and ES for risk aggregation}

\subsection{Robustness of risk measures}

The robustness of a statistical functional or an estimation procedure describes the sensitivity to underlying model deviations and/or data changes. Different definitions and interpretations of robustness exist in the literature; see for example Huber and Ronchetti (2009) from a pure statistical perspective, Hansen and Sargent (2007) in the context of economic decision making, and Ben-Tal et al. (2009) on robust optimization. In statistics, robustness mainly concerns the so-called distributional (or Hampel-Huber) robustness: the statistical consequences when the shape of the actual underlying distribution deviates slightly from the assumed model.

A risk measure $\rho$ is a function which maps a risk in $L^{0}$ to a number, $\rho: L^{0} \rightarrow \mathbb{R} \cup\{+\infty\}$. A risk measure is law-invariant if it only depends on the distribution of the risk. We omit the general introduction of risk measures, and refer the interested reader to Föllmer and Schied (2011). Since law-invariant risk measures are a specific type of statistical functionals, their robustness properties are already extensively studied in the statistical literature; see e.g. Huber and Ronchetti (2009).

In this paper, we focus on the two most popular risk measures: Value-at-Risk (VaR) at confidence level $p$, defined as

$$
\operatorname{VaR}_{p}(X)=\inf \{x \in \mathbb{R}: \mathbb{P}(X \leqslant x) \geqslant p\}, \quad p \in(0,1), X \in L^{0}
$$

and the Expected Shortfall (ES) at confidence level $p$, defined as

$$
\operatorname{ES}_{p}(X)=\frac{1}{1-p} \int_{p}^{1} \operatorname{VaR}_{q}(X) \mathrm{d} q, \quad p \in(0,1), X \in L^{0} .
$$

It is obvious that $\operatorname{VaR}_{p}(X)=F^{-1}(p)$ for $p \in(0,1)$ where $X \sim F$. Though typically in (2.2) it is assumed that $\mathbb{E}[|X|]<\infty$, we may occasionally allow that $\operatorname{ES}_{p}(X)=\infty$ for some $X$. On the other hand, $\operatorname{VaR}_{p}(X)$ is always a finite number for $X \in L^{0}$. 
It is often argued in the literature that quantile-based risk measures, such as VaR, are more robust as compared to mean-based risk measures, such as ES; the notion of robustness used most often is Hampel's (Hampel et al. (1986)). ES is only robust with respect to stronger metrics (e.g. the Wasserstein distance, Dobrushin (1970)); arguments of this type can be found in, for instance, Cont et al. (2010), Kou and Peng (2014) and Emmer et al. (2014). It is wellknown that the qualitative robustness of a statistical estimator, as in Hampel et al. (1986), is equivalent to the continuity of the corresponding risk measure at the true distribution. Thus, to analyze statistical robustness, one typically studies the continuity at distributions of a risk measure. Based on such consideration, we say that a law-invariant risk measure is robust at a distribution $F$ if it is continuous at $F$ in some metric. To be precise, $\rho$ is robust if for all $\varepsilon>0$, there exists $\delta>0$, such that $|\rho(X)-\rho(Y)|<\varepsilon$ whenever $d(F, G)<\delta$, where $d$ is some distance between the distributions $F$ and $G$ of $X$ and $Y$. For example, the Lévy distance in Huber and Ronchetti (2009) is used in Cont et al. (2010) to measure the difference between any two univariate distributions $F$ and $G$ :

$$
d(F, G):=\inf \{\varepsilon>0: F(x-\varepsilon)-\varepsilon<G(x)<F(x+\varepsilon)+\varepsilon, \quad \forall x \in \mathbb{R}\} .
$$

For rvs $S$ and $T$, we also denote $d(S, T):=d(F, G)$ where $F$ and $G$ are the distributions of $S$ and $T$, respectively. Note that the Lévy distance metrizes the weak topology on the set of distributions. Other metrics can also be used for the analysis of robustness; see Emmer et al. (2014). It was shown in Cont et al. (2010) that $\mathrm{VaR}_{p}$ is continuous at $F$ if $F^{-1}$ is continuous at $p$, while $\mathrm{ES}_{p}$ is not continuous at any distribution $F$. This result is often used to argue that $\mathrm{VaR}$ has better robustness properties than ES. Note that if $F^{-1}$ is not continuous at $p$, then $\mathrm{VaR}_{p}$ is not continuous at $F$ in the Lévy metric. In Krätschmer et al. (2014) it is argued that Hampel's notion of (statistical) robustness is less relevant for risk management. Using a different definition, they introduce a continuous scale of robustness.

In the following we will introduce a new, in our opinion practically relevant notion of robustness for risk aggregation, which favors ES over VaR.

\subsection{Aggregation-robustness}

In this section, we show that if model uncertainty lies at the level of the dependence structure but not at single risks, then VaR is not robust but ES is. For single risks $X_{i}, i=1, \ldots, n$, the aggregate risk $S$ is simply defined as $S=X_{1}+\cdots+X_{n}$. Often in practice, a joint model of $X_{1}, \ldots, X_{n}$ is modeled in two stages: $n$ marginal distributions $F_{1}, \ldots, F_{n}$ and a dependence structure (often through a copula $C$ ). As the modeling for marginal distributions is relatively easy, the dependence structure can be really difficult to model, statistically estimate and test. 
Considerable model uncertainty, which is often different in nature from the model uncertainty of marginal distributions, arises from modeling the dependence structure. In the following, we study robustness with respect to uncertainty in the dependence structure; for the purpose of this paper we assume the marginal distributions $F_{1}, \ldots, F_{n}$ are given.

When the dependence structure between the risks is unknown, the possible distributions of $S$ form a class of distributions. We denote the admissible class as

$$
\mathfrak{S}_{n}\left(F_{1}, \ldots, F_{n}\right)=\left\{X_{1}+\cdots+X_{n}: X_{i} \sim F_{i}, i=1, \ldots, n\right\},
$$

which for simplicity we further denote as $\mathfrak{S}_{n}=\mathfrak{S}_{n}\left(F_{1}, \ldots, F_{n}\right)$ if $\left(F_{1}, \ldots, F_{n}\right)$ is clear from the context. $\mathfrak{S}_{n}$ is the set of all possible aggregate risks. Note that for notational convenience, we left out portfolio weight factors; these can easily be reintroduced when necessary. Risk aggregation with dependence uncertainty concerns the probabilistic and statistical behavior of $S \in \mathfrak{S}_{n}$; see Bernard et al. (2014). We say that an admissible class is compatible with a risk measure $\rho$ if $\rho\left(X_{i}\right)<\infty$ and $\mathbb{E}\left[\left|X_{i}\right| \mathrm{I}_{\left\{X_{i}<0\right\}}\right]<\infty$ for $X_{i} \sim F_{i}, i=1, \ldots, n$ (the profit of each risk does not have an infinite mean; note that here we allow $\mathbb{E}\left[X_{i} \mathrm{I}_{\left\{X_{i} \geqslant 0\right\}}\right]=\infty$, i.e. extremely heavy-tailed risks).

Definition 2.1 (Aggregation-robustness). A law-invariant risk measure $\rho: L^{0} \rightarrow \mathbb{R} \cup\{+\infty\}$ is aggregation-robust, if for all $n \in \mathbb{N}$ and admissible classes $\mathfrak{S}_{n}$ compatible with $\rho$, and all $\varepsilon>0$, there exists $\delta>0$, such that $|\rho(S)-\rho(T)|<\varepsilon$, for all $S, T \in \mathfrak{S}_{n}$ such that $d(S, T)<\delta$.

The robustness character of Definition 2.1 in intuitively clear. If the joint distributions of $\left(X_{1}, \ldots, X_{n}\right)$ and $\left(Y_{1}, \ldots, Y_{n}\right)$ are close according to some metric, say, then the distributions of $X_{1}+\cdots+X_{n}$ and $Y_{1}+\cdots+Y_{n}$ are also close according to some metric. As a consequence, $\rho$ is insensitive to small perturbations of the joint distribution of the underlying risk factors, keeping the marginal distributions of the individual risks fixed. It is clear that Hampel's robustness, as discussed in Cont et al. (2010), without the restriction of $S, T$ being in a common admissible class, implies aggregation-robustness. When the dependence structure is modeled by copulas, our definition of robustness implies that a risk measure is insensitive to the copula of the individual risks when the marginal distributions are assumed to be known. The fact that in Definition 2.1 we look at $S, T \in \mathfrak{S}_{n}$ reflects our interest in aggregation and diversification. One could of course look at other functional-robustness definitions beyond aggregation (summation).

Example 2.1 (VaR is not aggregation-robust). For $t \in[0,1]$, let $X_{t}$ and $Y_{t}$ have joint distribution $C_{t}$,

$C_{t}(x, y)=t x y+(1-t)(\max \{\min \{x, 1 / 2\}+\min \{y, 1 / 2\}-1 / 2,0\}+\max \{x+y-3 / 2,0\}), x, y \in[0,1]$. 
It is easy to see that $X_{t}$ and $Y_{t}$ are both $\mathrm{U}[0,1]$ distributed, hence $C_{t}$ is a copula, $t \in[0,1]$. Note that $C_{t}, t \in(0,1)$ is a mixture of the independent copula $C_{1}$ and another copula

$$
C_{0}:[0,1]^{2} \rightarrow[0,1],(x, y) \mapsto \max \{\min \{x, 1 / 2\}+\min \{y, 1 / 2\}-1 / 2,0\}+\max \{x+y-3 / 2,0\}
$$

$C_{0}$ is the ordinal sum of two Fréchet lower copulas; see Neslen (2006, Section 3.2.2).

It is immediate that the distribution of $X_{t}+Y_{t}$ for $t \in(0,1]$ is symmetric, centered at 1 , with positive density on the interval $(1 / 2,3 / 2)$. Thus, $\operatorname{VaR}_{1 / 2}\left(X_{t}+Y_{t}\right)=1$. It is also straightforward that $X_{0}+Y_{0}$ is a degenerate rv on $\{1 / 2,3 / 2\}$ with $\operatorname{VaR}_{1 / 2}\left(X_{0}+Y_{0}\right)=1 / 2$. As a consequence, $\operatorname{VaR}_{1 / 2}\left(X_{0}+Y_{0}\right) \neq \lim _{t \rightarrow 0} \operatorname{VaR}_{1 / 2}\left(X_{t}+Y_{t}\right)$. Based on the simple fact that $d\left(X_{t}+Y_{t}, X_{0}+Y_{0}\right) \rightarrow 0$ as $t$ goes to zero, we conclude that $\mathrm{VaR}_{1 / 2}$ is not aggregation-robust. A similar example can be constructed for $\operatorname{VaR}_{p}, p \in(0,1)$.

Note that in the above example, the joint distribution $C_{t}$ with a small $t>0$ can be seen as the joint distribution $C_{0}$ influenced by a small perturbation. It is moreover worth noting that in Example 2.1, the marginal distributions are continuous with positive densities. Hence, even if the true marginal distributions are known to have positive densities, VaR can still be discontinuous in aggregation. This observation weakens the robustness of VaR in aggregation. On the other hand, we will see that ES is aggregation-robust, although it is well-known to be non-robust in Hampel's sense since it is discontinuous at any distribution with respect to the weak topology.

For generality, we study the aggregation-robustness of distortion risk measures, defined as

$$
\rho(X)=\int_{0}^{1} \operatorname{VaR}_{t}(X) \mathrm{d} h(t), \quad X \in L^{0} \text { such that the integeral is well-defined, }
$$

where $h$ is a probability measure on $(0,1] ; h$ is called the distortion function of $\rho$. See Wang et al. (1997) for distortion risk measures in the context of insurance premium calculations, Kusuoka (2001) for their connection with coherent risk measures, and Cont et al. (2010) for their robustness properties. A distortion risk measure $\rho$ is coherent if and only if $h$ is convex, in which case $\rho$ is called a spectral risk measure; see Acerbi (2002). Distortion risk measures are also closely related to $L$-statistics; see Huber and Ronchetti (2009, Section 3.3). For $p \in(0,1), \operatorname{VaR}_{p}$ and $\mathrm{ES}_{p}$ are special cases of distortion risk measures, with distortion functions $h(t)=\mathrm{I}_{\{t \geqslant p\}}, t \in[0,1]$ and $h(t)=\mathrm{I}_{\{t \geqslant p\}}(t-p) /(1-p), t \in[0,1]$, respectively. Our main result on robustness now becomes:

Theorem 2.1. All coherent distortion risk measures are aggregation-robust.

The main contribution of Theorem 2.1 is to justify that, when the model uncertainty lies at the level of dependence but not at the level of the marginal distributions, coherent risk measures, such as ES, are more robust than VaR. 
Remark 2.1. Cont et al. (2010) also introduced the notion of $\mathcal{C}$-robustness, where $\mathcal{C}$ is a set of distributions. A risk measure $\rho$ is $\mathcal{C}$-robustness if $\rho$ is continuous in $\mathcal{C}$ with respect to the Lévy distance; see Cont et al. (2010, Proposition 2). Using this notion, $\operatorname{VaR}_{p}$ is $\mathcal{C}_{p}$-robust, where $\mathcal{C}_{p}$ is the set of distributions $F$ for which $F^{-1}$ is continuous at $p$. If we denote by $\mathfrak{D}\left(\mathfrak{S}_{n}\right)$ the set of all possible distributions of an admissible class $\mathfrak{S}_{n}$, then $\rho$ is aggregation-robust if and only if $\rho$ is $\mathfrak{D}\left(\mathfrak{S}_{n}\right)$-robust for all possible choices of $n \in \mathbb{N}$ and $\mathfrak{D}\left(\mathfrak{S}_{n}\right)$, in which $\mathfrak{S}_{n}$ is compatible with $\rho$.

Our result can be interpreted using weak convergence in the admissible class $\mathfrak{S}_{n}$. For $S, S_{1}, S_{2}, \cdots \in \mathfrak{S}_{n}$ and $S_{k} \rightarrow S$ weakly as $k \rightarrow \infty$, we have that $\operatorname{ES}_{p}\left(S_{k}\right) \rightarrow \operatorname{ES}_{p}(S)$ as $k \rightarrow \infty$ by Theorem 2.1. As illustrated by Example 2.1, the convergence $\operatorname{VaR}_{p}\left(S_{k}\right) \rightarrow \operatorname{VaR}_{p}(S)$ as $k \rightarrow \infty$ may fail to hold.

Finally, we remark that it would be of much interest to characterize the class of aggregationrobust statistical functionals (risk measures). Such a characterization is beyond the scope of this paper and we leave it for future work.

\section{Bounds on VaR aggregation}

Given the considerable statistical difficulty in modeling inter-dependency of risks, one may worry about, in addition to the sensitivity of risk measures to a small change in the dependence structure as discussed in Section 2, the uncertainty of a misspecified dependence structure which is relatively far away from that of the true underlying portfolio. In this section, we study the worst-case values of VaR when the dependence structure in a portfolio with given marginal distributions is unknown. These values can be used to analyze extreme scenarios of risk aggregation and determine conservative capital requirement under model uncertainty.

\subsection{Aggregation and diversification under dependence uncertainty}

We start with the motivating notion of diversification ratio, which is closely related to the aggregation of VaR. Given a portfolio consisting of individual risks $X_{1}, \ldots, X_{n}$, the diversification ratio of VaR at confidence level $p \in(0,1)$ is defined as

$$
\Delta_{n}^{p}=\frac{\operatorname{VaR}_{p}\left(X_{1}+\cdots+X_{n}\right)}{\sum_{i=1}^{n} \operatorname{VaR}_{p}\left(X_{i}\right)} .
$$

The diversification ratio measures a kind of diversification benefit, and is for instance widely used in operational risk (see examples in Embrechts et al. (2013)). In the latter context, $X_{i}$ corresponds to next year's operational risk loss in business line $i, i=1, \ldots, n$; often explicit models for the loss-dependence among business lines are not available. For capital charge purposes, one estimates the total capital requirement for the superposition of the risks in each business line. 
One then typically adds up the risk measures across all business lines, and multiplies by a factor which is an estimate of $\Delta_{n}^{p}$. For this purpose, one needs a joint model of the risks $X_{1}, \ldots, X_{n}$.

With a known joint distribution of $\left(X_{1}, \ldots, X_{n}\right), \Delta_{n}^{p}$ may be calculated theoretically. If $\Delta_{n}^{p} \leqslant 1$, we say there is a diversification benefit in the portfolio; if $\Delta_{n}^{p} \geqslant 1$, we say there is a diversification penalty in the portfolio. When $F_{1}, \ldots, F_{n}$ are known and the joint model of $\left(X_{1}, \ldots, X_{n}\right)$ is unspecified, the worst diversification ratio is defined as

$$
\bar{\Delta}_{n}^{p}=\frac{\sup \left\{\operatorname{VaR}_{p}\left(X_{1}+\cdots+X_{n}\right): X_{i} \sim F_{i}, i=1, \ldots, n\right\}}{\sum_{i=1}^{n} \operatorname{VaR}_{p}\left(X_{i}\right)}=\frac{\sup \left\{\operatorname{VaR}_{p}(S): S \in \mathfrak{S}_{n}\right\}}{\sum_{i=1}^{n} \operatorname{VaR}_{p}\left(X_{i}\right)} .
$$

By definition $\bar{\Delta}_{n}^{p} \geqslant 1$ if $\sum_{i=1}^{n} \operatorname{VaR}_{p}\left(X_{i}\right)>0$. In the following we denote the comonotonic VaR by $\operatorname{VaR}_{p}^{+}\left(S_{n}\right)$, i.e.

$$
\operatorname{VaR}_{p}^{+}\left(S_{n}\right)=\sum_{i=1}^{n} \operatorname{VaR}_{p}\left(X_{i}\right)
$$

Note here that $S_{n}$ is symbolic and does not represent a particular rv. The calculation of $\bar{\Delta}_{n}^{p}$, as a measure of the worst-case diversification effect of VaR, serves two purposes:

- Conservative capital requirement. $\bar{\Delta}_{n}^{p} \operatorname{VaR}_{p}^{+}\left(S_{n}\right)$ can be used as the most conservative capital requirement in the case of given (or estimated) marginal distributions $F_{1}, \ldots, F_{n}$ of the individual risks.

- Measurement of model uncertainty. If $\bar{\Delta}_{n}^{p}$ is small, then the model uncertainty is small, and the risk measure $\mathrm{VaR}$ is considered as less problematic in risk aggregation; capital requirement principles based on $\mathrm{VaR}_{p}^{+}$become more plausible. If $\bar{\Delta}_{n}^{p}$ is large, then the model uncertainty is severe, and arguments of diversification benefit need to be taken with care.

The best diversification ratio, replacing the sup by an inf, can be studied similarly. Since we are more interested in the worst-case (corresponding to a conservative capital requirement), we omit a discussion of the best diversification ratio.

In the recent literature, it was shown that the value of $\bar{\Delta}_{n}^{p}$ is closely related to the risk measure ES. Denote the worst-case ES by $\overline{\operatorname{ES}}_{p}\left(S_{n}\right)=\sup \left\{\operatorname{ES}_{p}(S): S \in \mathfrak{S}_{n}\right\}$; since ES is subadditive and comonotonic additive, we have that

$$
\overline{\operatorname{ES}}_{p}\left(S_{n}\right)=\sum_{i=1}^{n} \operatorname{ES}_{p}\left(X_{i}\right)=\operatorname{ES}_{p}^{+}\left(S_{n}\right),
$$

where the latter +-notation is in line with the notation used for the comonotonic VaR case. Since VaR is bounded by ES, the worst-case VaR is bounded by the worst-case ES. If $\operatorname{VaR}_{p}^{+}\left(S_{n}\right)>0$, we have the following direct upper bound for $\bar{\Delta}_{n}^{p}$ :

$$
1 \leqslant \bar{\Delta}_{n}^{p} \leqslant \frac{\operatorname{ES}_{p}^{+}\left(S_{n}\right)}{\operatorname{VaR}_{p}^{+}\left(S_{n}\right)}=\frac{\overline{\operatorname{ES}}_{p}\left(S_{n}\right)}{\operatorname{VaR}_{p}^{+}\left(S_{n}\right)} .
$$


See also Embrechts et al. (2014) for a discussion on this upper bound. Later in this section we will show that the second inequality in (3.1) is asymptotically sharp as $n \rightarrow \infty$.

By definition, calculation of the worst diversification ratio is equivalent to the calculation of the worst-case VaR

$$
\overline{\operatorname{VaR}}_{p}\left(S_{n}\right):=\sup \left\{\operatorname{VaR}_{p}(S): S \in \mathfrak{S}_{n}\right\}
$$

For the history and a general discussion on problems related to (3.2) from the perspective of quantitative risk management, we refer to Embrechts et al. (2014). When $F_{1}=F_{2}=\cdots=$ $F_{n}=: F$, i.e. the homogeneous case, Wang et al. (2013) obtained $\overline{\operatorname{VaR}}_{p}\left(S_{n}\right)$ for $F$ with a taildecreasing density. If $F_{1}, \ldots, F_{n}$ are not identical, explicit calculations of $\overline{\operatorname{VaR}}_{p}\left(S_{n}\right)$ and $\bar{\Delta}_{n}^{p}$ are not available in general. Embrechts et al. (2013) introduced the Rearrangement Algorithm to numerically calculate $\overline{\operatorname{VaR}}_{p}\left(S_{n}\right)$ based on a discretized approximation.

Regarding the asymptotic behavior of $\overline{\operatorname{VaR}}_{p}\left(S_{n}\right)$ and $\bar{\Delta}_{n}^{p}$, Puccetti and Rüschendorf (2014) obtained that, as $n \rightarrow \infty$,

$$
\frac{\overline{\operatorname{VaR}}_{p}\left(S_{n}\right)}{\overline{\operatorname{ES}}_{p}\left(S_{n}\right)} \rightarrow 1
$$

in the homogeneous case under a condition of complete mixability for the marginal distributions. See also Wang (2014) and Wang and Wang (2014) for weaker conditions so that (3.3) holds. Puccetti et al. (2013) considered the case when there are finitely many different marginal distributions in the sequence $F_{1}, F_{2}, \ldots$ and obtained the same equivalence (3.3). A consequence of (3.3) is that

$$
\lim _{n \rightarrow \infty} \bar{\Delta}_{n}^{p}=\lim _{n \rightarrow \infty} \frac{\overline{\operatorname{ES}}_{p}\left(S_{n}\right)}{\operatorname{VaR}_{p}^{+}\left(S_{n}\right)},
$$

given that the right-hand limit exists. That is, the second inequality in (3.1) is asymptotically sharp. However, as mentioned above, the existing results only deal with the (almost) homogeneous case, and some specific assumptions on the marginal distributions need to be imposed. Later in this section, we will provide analytical approximations for $\overline{\operatorname{VaR}}_{p}\left(S_{n}\right)$ and $\bar{\Delta}_{n}^{p}$. Based on these results, we will give a proof of (3.3) and (3.4) under very general conditions and, moreover, obtain a rate of convergence.

\subsection{Bounds on VaR aggregation for a finite number of risks}

In this section, we will give inequalities for the worst-case and best-case VaR and its diversification ratio. For the rest of Sections 3 and 4 , we assume that the distributions $F_{i}, i \in \mathbb{N}$ are continuous; this constitutes no real restriction, and is only assumed for the ease of notation. For a continuous distribution $F_{i}$, define

$$
\mu_{p, q}^{(i)}=\frac{1}{q-p} \int_{p}^{q} F_{i}^{-1}(t) \mathrm{d} t
$$


for $1 \geqslant q>p \geqslant 0, i=1, \ldots, n$. Note that $\mu_{0, q}^{(i)}$ and $\mu_{p, 1}^{(i)}$ might be infinite. Using the above notation, it is immediate that

$$
\overline{\operatorname{ES}}_{p}\left(S_{n}\right)=\sum_{i=1}^{n} \operatorname{ES}_{p}\left(X_{i}\right)=\sum_{i=1}^{n} \mu_{p, 1}^{(i)} .
$$

For future discussion, we also denote the best-case $\operatorname{VaR}$ by $\underline{\operatorname{VaR}}_{p}\left(S_{n}\right)$, that is

$$
\underline{\operatorname{VaR}}_{p}\left(S_{n}\right)=\inf _{S \in \mathfrak{S}_{n}} \operatorname{VaR}_{p}(S)
$$

and the best-case ES by $\underline{\operatorname{ES}}_{p}\left(S_{n}\right)$, that is

$$
\underline{\mathrm{ES}}_{p}\left(S_{n}\right)=\inf _{S \in \mathfrak{S}_{n}} \operatorname{ES}_{p}(S)
$$

Analytical formulas for each of $\overline{\operatorname{VaR}}_{p}\left(S_{n}\right), \underline{\operatorname{VaR}}_{p}\left(S_{n}\right)$ and $\underline{\mathrm{ES}}_{p}\left(S_{n}\right)$ are not available under general assumptions on the marginal distributions; see Bernard et al. (2014) and Embrechts et al. (2014) for existing results on $\overline{\operatorname{VaR}}_{p}\left(S_{n}\right), \underline{\operatorname{VaR}}_{p}\left(S_{n}\right)$ and $\underline{\mathrm{ES}}_{p}\left(S_{n}\right)$.

The following theorem contains our main result regarding approximations of $\overline{\operatorname{VaR}}_{p}\left(S_{n}\right)$ and $\underline{\operatorname{VaR}}_{p}\left(S_{n}\right)$.

Theorem 3.1. Suppose $F_{1}, \ldots, F_{n}$ are continuous distributions. We have for $p \in(0,1)$,

$$
\sup _{q \in(p, 1]}\left\{\sum_{i=1}^{n} \mu_{p, q}^{(i)}-\max _{i=1, \ldots, n}\left(F_{i}^{-1}(q)-F_{i}^{-1}(p)\right)\right\} \leqslant \overline{\operatorname{VaR}}_{p}\left(S_{n}\right) \leqslant \overline{\operatorname{ES}}_{p}\left(S_{n}\right),
$$

and

$$
\sum_{i=1}^{n} \mu_{0, p}^{(i)} \leqslant \underline{\operatorname{VaR}}_{p}\left(S_{n}\right) \leqslant \inf _{q \in[0, p)}\left\{\sum_{i=1}^{n} \mu_{q, p}^{(i)}+\max _{i=1, \ldots, n}\left(F_{i}^{-1}(q)-F_{i}^{-1}(p)\right)\right\} .
$$

In particular, if $F_{1}, \ldots, F_{n}$ are supported on $[a, b], a<b, a, b \in \mathbb{R}$, then

$$
\overline{\mathrm{ES}}_{p}\left(S_{n}\right)-(b-a) \leqslant \overline{\operatorname{VaR}}_{p}\left(S_{n}\right) \leqslant \overline{\mathrm{ES}}_{p}\left(S_{n}\right) .
$$

Note that in the case when all marginal distributions are bounded, $\overline{\operatorname{VaR}}_{p}\left(S_{n}\right)$ and $\overline{\operatorname{ES}}_{p}\left(S_{n}\right)$ differ by at most a constant which does not depend on $n$. Theorem 3.1 can also be formulated for the worst diversification ratio of VaR.

Corollary 3.2. Suppose $F_{1}, \ldots, F_{n}$ are continuous distributions, and $\operatorname{VaR}_{p}^{+}\left(S_{n}\right)>0$. We have for $p \in(0,1)$,

$$
\sup _{q \in(p, 1]}\left\{\frac{\sum_{i=1}^{n} \mu_{p, q}^{(i)}-\max _{i=1, \ldots, n}\left(F_{i}^{-1}(q)-F_{i}^{-1}(p)\right)}{\operatorname{VaR}_{p}^{+}\left(S_{n}\right)}\right\} \leqslant \bar{\Delta}_{n}^{p} \leqslant \frac{\overline{\mathrm{ES}}_{p}\left(S_{n}\right)}{\operatorname{VaR}_{p}^{+}\left(S_{n}\right)} .
$$

In particular, if $F_{1}, \ldots, F_{n}$ are supported in $[a, b], a<b, a, b \in \mathbb{R}$, then

$$
\frac{\overline{\mathrm{ES}}_{p}\left(S_{n}\right)}{\operatorname{VaR}_{p}^{+}\left(S_{n}\right)}-\frac{b-a}{\operatorname{VaR}_{p}^{+}\left(S_{n}\right)} \leqslant \bar{\Delta}_{n}^{p} \leqslant \frac{\overline{\mathrm{ES}}_{p}\left(S_{n}\right)}{\operatorname{VaR}_{p}^{+}\left(S_{n}\right)}
$$


In the homogeneous case, i.e. $F:=F_{1}=F_{2}=\ldots$, the left-hand side and right-hand side of (3.9) both converge to $\frac{\mathrm{ES}_{p}(X)}{\operatorname{VaR}_{p}(X)}$ as $n \rightarrow \infty$, where $X \sim F$, assuming $\operatorname{VaR}_{p}(X) \neq 0$. In the following, we will study the limit of the worst- and best-case VaR and its diversification ratio under general marginal assumptions, as $n$ goes to infinity.

\subsection{Asymptotic equivalence and limit of the worst diversification ratio}

Based on Theorem 3.1, we now derive the asymptotic equivalence between the worst-case VaR and the worst-case ES under very weak general conditions. For an asymptotic analysis, some uniformality conditions on $F_{i}, i \in \mathbb{N}$ need to be imposed. In what follows, $X_{i}$ is any rv with distribution $F_{i}, i \in \mathbb{N}$. Define the following conditions, for some $p \in(0,1)$ and $k>1$ :

(a) $\mathbb{E}\left[\left|X_{i}-\mathbb{E}\left[X_{i}\right]\right|^{k}\right]<M$ for some $M>0$

(b) $\liminf _{n \rightarrow \infty} n^{-1 / k} \sum_{i=1}^{n} \operatorname{ES}_{p}\left(X_{i}\right)=+\infty$, and

$\left(\mathrm{b}^{*}\right) C_{0}:=\liminf _{n \rightarrow \infty} \frac{1}{n} \sum_{i=1}^{n} \operatorname{ES}_{p}\left(X_{i}\right)>0$.

The above conditions only concern the moments of $F_{i}, i \in \mathbb{N}$, and hence they are quite weak and commonly satisfied. Condition (a) is a uniform boundedness condition, ensuring that the aggregate portfolio $S_{n}$ does not contain a single risk with a too heavy tail that dominates the other risks. Condition (b) is assumed to guarantee that the average ES of the sequence of risks does not vanish to zero too fast. Without (a) or (b), the limiting portfolio would exhibit a finite- $n$ behavior. Hence, in view of an asymptotic analysis, both conditions are reasonable. The condition $\left(\mathrm{b}^{*}\right)$ is a stronger version of (b). In particular, in the homogeneous case when $F_{i}$, $i \in \mathbb{N}$ are identical, $\operatorname{ES}_{p}\left(X_{1}\right)>0$ implies (b*) and hence it also implies (b). We also remark that condition $\left(\mathrm{a}^{*}\right)$ below is stronger than condition $(\mathrm{a})$ :

$\left(\mathrm{a}^{*}\right) \mathbb{E}\left[\left|X_{i}\right|^{k}\right]$ is uniformly bounded.

Theorem 3.3. Suppose that the continuous distributions $F_{i}, i \in \mathbb{N}$, satisfy (a) and (b) for some $p \in(0,1)$ and $k>1$, then

$$
\lim _{n \rightarrow \infty} \frac{\overline{\operatorname{VaR}}_{p}\left(S_{n}\right)}{\overline{\operatorname{ES}}_{p}\left(S_{n}\right)}=1 .
$$

If, in addition, (b) is replaced by $\left(b^{*}\right)$, then for sufficiently large $n$,

$$
1 \geqslant \frac{\overline{\operatorname{VaR}}_{p}\left(S_{n}\right)}{\overline{\operatorname{ES}}_{p}\left(S_{n}\right)} \geqslant 1-C n^{-1+1 / k},
$$

where

$$
C=\left(\frac{1}{1-p} \frac{k}{k-1}+1\right) \frac{M^{1 / k}}{C_{0}}>0,
$$

$M$ is given in (a) and $C_{0}$ is given in $\left(b^{*}\right)$. 
Theorem 3.3 establishes the asymptotic equivalence of the worst-case ES and the worstcase VaR for risk aggregation for general, possibly inhomogeneous portfolios. As mentioned in Section 3.1, homogeneous or almost-homogeneous cases for (3.10) to hold were previously obtained in the literature. While existing methods of proof were mainly based on the theory of complete mixability, an extension using the same techniques to arbitrarily many different marginal distributions was not possible.

Similarly to Theorem 3.3, we can obtain the limit of the best-case VaR bounds. In the following we define the left-tail ES (LES) as

$$
\operatorname{LES}_{p}(X)=\frac{1}{p} \int_{0}^{p} \operatorname{VaR}_{q}(X) \mathrm{d} q=-\operatorname{ES}_{p}(-X),
$$

and denote its best-case value under dependence uncertainty by

$$
\underline{\operatorname{LES}}_{p}\left(S_{n}\right):=\inf _{S \in \mathfrak{S}_{n}} \operatorname{LES}_{p}(S)=\sum_{i=1}^{n} \operatorname{LES}_{p}\left(X_{i}\right)=\sum_{i=1}^{n} \mu_{0, p}^{(i)},
$$

where the second equality can be seen from the symmetry between ES and LES. For the best-case VaR bounds, we use a slightly different set of conditions, for some $p \in(0,1)$ and $k>1$ :

(c) $\liminf n_{n \rightarrow \infty} n^{-1 / k} \sum_{i=1}^{n} \operatorname{LES}_{p}\left(X_{i}\right)=+\infty$, and

(c*) $C_{0}:=\liminf _{n \rightarrow \infty} \frac{1}{n} \sum_{i=1}^{n} \operatorname{LES}_{p}\left(X_{i}\right)>0$.

The following corollary is obtained from Theorem 3.3 by symmetry.

Corollary 3.4. Suppose that the continuous distributions $F_{i}, i \in \mathbb{N}$, satisfy (a) and (c) for some $p \in(0,1)$ and $k>1$, then

$$
\lim _{n \rightarrow \infty} \frac{\operatorname{VaR}_{p}\left(S_{n}\right)}{\underline{\operatorname{LES}}_{p}\left(S_{n}\right)}=1 .
$$

If, in addition, (c) is replaced by $\left(c^{*}\right)$, then for sufficiently large $n$,

$$
1 \geqslant \frac{\operatorname{VaR}_{p}\left(S_{n}\right)}{\underline{\operatorname{LES}}_{p}\left(S_{n}\right)} \geqslant 1-C n^{-1+1 / k},
$$

where

$$
C=\left(\frac{1}{1-p} \frac{k}{k-1}+1\right) \frac{M^{1 / k}}{C_{0}}>0,
$$

$M$ is given in (a) and $C_{0}$ is given in $\left(c^{*}\right)$.

Remark 3.1. The conditions (c) and $\left(\mathrm{c}^{*}\right)$ are slightly stronger than $(\mathrm{b})$ and $\left(\mathrm{b}^{*}\right)$, respectively, and this asymmetry is due to the fact that we mainly consider the cases when the aggregate risk measures LTE and ES are positive. The asymmetry can be trivially removed by assuming $\liminf _{n \rightarrow \infty}\left|\frac{1}{n} \sum_{i=1}^{n} \operatorname{LES}_{p}\left(X_{i}\right)\right|>C_{0}$ instead of (c). 
Finally, we remark that the limit of $\bar{\Delta}_{n}^{p}$ as $n \rightarrow \infty$ can be obtained directly from Theorem 3.3. Suppose the continuous distributions $F_{i}, i \in \mathbb{N}$ satisfy (a) and (b) for some $p \in(0,1)$ and $k>1$, then, as $n \rightarrow \infty$,

$$
\bar{\Delta}_{n}^{p} \frac{\operatorname{VaR}_{p}^{+}\left(S_{n}\right)}{\overline{\operatorname{ES}}_{p}\left(S_{n}\right)} \rightarrow 1
$$

If in addition, $R_{p}:=\lim _{n \rightarrow \infty} \frac{\overline{\operatorname{ES}}_{p}\left(S_{n}\right)}{\operatorname{VaR}_{p}^{+}\left(S_{n}\right)}$ exists in $[1, \infty]$, then $\Delta_{n}^{p} \rightarrow R_{p}$ as $n \rightarrow \infty$.

\section{Uncertainty spread of VaR and ES}

In addition to the distribution-wise continuity as discussed in Section 2, in this section we study the uncertainty spread of VaR and ES when the dependence structure is completely unknown, based on results obtained in Section 3. This quantifies the magnitude of dependence uncertainty in a model for risk aggregation. We show that VaR generally exhibits a larger spread compared to ES. This result suggests that VaR is more sensitive to dependence uncertainty compared to ES and can be seen as a supporting argument for Theorem 2.1. For $p \in(0,1)$ we define the dependence uncertainty spread (DU-spread) of $\mathrm{VaR}_{p}$ as

$$
\overline{\operatorname{VaR}}_{p}\left(S_{n}\right)-\underline{\operatorname{VaR}}_{p}\left(S_{n}\right),
$$

and of $\mathrm{ES}_{p}$ as

$$
\overline{\mathrm{ES}}_{p}\left(S_{n}\right)-\underline{\mathrm{ES}}_{p}\left(S_{n}\right)
$$

See Embrechts et al. (2014) for a discussion on the DU-spread of VaR and its relevance in risk management.

By definition $\operatorname{ES}_{p}(X) \geqslant \operatorname{VaR}_{p}(X)$ for any risk $X$ and the inequality is strict when $X$ is continuous. Naturally, when switching from VaR to ES for the purpose of capital requirement, one should consider a lower confidence level for ES. In the most recent consultative document BCBS (2013), it was proposed that for internal risk models, $\mathrm{VaR}_{0.99}$ should be replaced by $\mathrm{ES}_{0.975}$ which often yields a similar value to $\mathrm{VaR}_{0,99}$ for light-tailed risks. Under the Swiss Solvency Test (SST), $\mathrm{VaR}_{0.995}$ is used to compare with $\mathrm{ES}_{0.99}$ to calculate the capital requirement for the change in the Risk Bearing Capital (RBC) over a one-year period; see (EIOPA, 2011, p.32). Kou and Peng (2014) also proposed that, in order to compare with $\mathrm{ES}_{p}$, one could use the corresponding Median Shortfall (MS), which is the median of the conditional tail distribution above $\mathrm{VaR}_{p}$, and hence satisfies

$$
\operatorname{MS}_{p}(X)=\operatorname{VaR}_{(p+1) / 2}(X) ;
$$

thus, it is consistent with the SST regime. Hence, it may be useful to compare the DU-spread of $\mathrm{VaR}_{q}$ and $\mathrm{ES}_{p}$ for $q \geqslant p$. The following proposition compares the DU-spread of $\mathrm{VaR}_{q}$ and that 
of $\mathrm{ES}_{p}$ in the asymptotic sense. In what follows, we denote by $\mu_{n}$ the summation of the means of $F_{1}, \ldots, F_{n}$, assumed to exist.

Proposition 4.1. Suppose $1>q \geqslant p>0$.

(i) Suppose that the continuous distributions $F_{i}, i \in \mathbb{N}$, satisfy (a) and (c), then

$$
\liminf _{n \rightarrow \infty} \frac{\overline{\operatorname{VaR}}_{q}\left(S_{n}\right)-\underline{\operatorname{VaR}}_{q}\left(S_{n}\right)}{\overline{\mathrm{ES}}_{p}\left(S_{n}\right)-\underline{\mathrm{ES}}_{p}\left(S_{n}\right)}=\liminf _{n \rightarrow \infty} \frac{\overline{\mathrm{ES}}_{q}\left(S_{n}\right)-\underline{\mathrm{LES}}_{q}\left(S_{n}\right)}{\overline{\mathrm{ES}}_{p}\left(S_{n}\right)-\underline{\mathrm{ES}}_{p}\left(S_{n}\right)} \geqslant \liminf _{n \rightarrow \infty} \frac{\overline{\operatorname{ES}}_{q}\left(S_{n}\right)-\mu_{n}}{\overline{\mathrm{ES}}_{p}\left(S_{n}\right)-\mu_{n}} \geqslant 1 .
$$

(ii) Suppose that the distributions $F_{i}, i \in \mathbb{N}$, are identical and equal to $F$, and $\mathbb{E}[|X|]<\infty$ where $X \sim F$, then

$$
\lim _{n \rightarrow \infty} \frac{\overline{\operatorname{VaR}}_{q}\left(S_{n}\right)-\underline{\operatorname{VaR}}_{q}\left(S_{n}\right)}{\overline{\operatorname{ES}}_{p}\left(S_{n}\right)-\underline{\mathrm{ES}}_{p}\left(S_{n}\right)}=\frac{\mathrm{ES}_{q}(X)-\mathrm{LES}_{q}(X)}{\operatorname{ES}_{p}(X)-\mathbb{E}[X]} \geqslant 1 .
$$

Proposition 4.1 suggests that VaR is overall more sensitive to dependence uncertainty for large $n$, compared to ES. Numerical evidence of the comparison of DU-spread for VaR and ES at the same level can be found in Section 5, even for small numbers of $n$. Note that, although the DU-spread of ES is smaller than that of VaR asymptotically, both risk measures have a rather large uncertainty spread in general, suggesting that dependence uncertainty in risk aggregation must be taken with care no matter whether ES or VaR is chosen as the underlying risk measure. Remark 4.1. In the homogeneous case, for any continuous distribution $F$, the limit of the DUspread ratio in (4.2) is strictly greater than 1 since $\operatorname{LES}_{q}(X)<\mathbb{E}[X]$ and $\operatorname{ES}_{q}(X)>\operatorname{ES}_{p}(X)$. In the case $q=p$, we note that, for light-tailed risks $X, \operatorname{LES}_{p}(X)$ is slightly smaller than $\mathbb{E}[X]$; for heavy-tailed risks $X, \operatorname{LES}_{p}(X)$ can be significantly smaller than $\mathbb{E}[X]$, leading to a much larger DU-spread of VaR. From Proposition 4.1, we can also see that, approximately, the $\mathrm{VaR}_{q}$ interval under DU is $\left[\sum_{i=1}^{n} \operatorname{LES}_{q}\left(X_{i}\right), \sum_{i=1}^{n} \mathrm{ES}_{q}\left(X_{i}\right)\right]$ and the $\mathrm{ES}_{p}$ interval under DU is $\left[\mu_{n}, \sum_{i=1}^{n} \mathrm{ES}_{p}\left(X_{i}\right)\right]$. Note that when $F_{i}, i \in \mathbb{N}$ are the same, no conditions other than a finite first moment of $F$ are needed for the asymptotic equivalence in Theorem 3.3 and Corollary 3.4 to hold. This was also suggested by Corollary 3.7 of Wang and Wang (2014). In this case, the limit of the ratio between the two uncertainty spreads is explicitly given in (4.2).

In the following we give a result for finite $n$, in the case of bounded risks. A proof can be directly obtained from Theorem 3.1.

Corollary 4.2. Suppose that $1>q \geqslant p>0, F_{1}, \ldots, F_{n}$ are continuous distributions, supported in $[a, b], a<b, a, b \in \mathbb{R}$, and

$$
\sum_{i=1}^{n}\left(\mathrm{ES}_{q}\left(X_{i}\right)+\mathbb{E}\left[X_{i}\right]-\mathrm{ES}_{p}\left(X_{i}\right)-\operatorname{LES}_{q}\left(X_{i}\right)\right)>2(b-a),
$$


where $X_{i} \sim F_{i}, i=1, \ldots, n$, then

$$
\frac{\overline{\operatorname{VaR}}_{q}\left(S_{n}\right)-\underline{\operatorname{VaR}}_{q}\left(S_{n}\right)}{\overline{\mathrm{ES}}_{p}\left(S_{n}\right)-\underline{\mathrm{ES}}_{p}\left(S_{n}\right)}>1
$$

Note that in Corollary 4.2, since $\operatorname{ES}_{q}\left(X_{i}\right) \geqslant \operatorname{ES}_{p}\left(X_{i}\right)$ and $\mathbb{E}\left[X_{i}\right] \geqslant \operatorname{LES}_{q}\left(X_{i}\right)$, the left-hand side of (4.3) is the summation of $n$ non-negative terms while the right-hand side of (4.3) is a constant, hence (4.3) holds for $n$ sufficiently large as long as the summation of the left-hand side of (4.3) diverges as $n \rightarrow \infty$.

We remark that it remains theoretically unclear whether the DU-spread of $\mathrm{VaR}_{q}$ is always larger than (or equal to) $\mathrm{ES}_{p}$ for finite $n$ and $q \geqslant p$. In all our numerical examples (see Section 5 below), $\mathrm{VaR}_{q}$ always has a larger DU-spread than $\mathrm{ES}_{p}$.

\section{Numerical examples}

As suggested by BCBS (2013), the risk measure $\mathrm{ES}_{0.975}$ is a candidate to replace $\mathrm{VaR}_{0.99}$. The SST (see EIOPA, 2011) used $\operatorname{VaR}_{(1+p) / 2}$ to compare with $\mathrm{ES}_{p}$. Based on such considerations, in this section, we provide the worst-case and the best-case values of $\mathrm{VaR}_{0.99}, \mathrm{VaR}_{0.9875}, \mathrm{VaR}_{0.975}$ and $\mathrm{ES}_{0.975}$ for different portfolios under dependence uncertainty. We compare the dependence uncertainty spread of VaR and ES in each model, and also look at the influence on the number $n$ of risks in the portfolio. The numerical calculation is carried out through the Rearrangement Algorithm (RA) described in Embrechts et al. (2013), with discretization step $\Delta x=10^{-6}$. The following three models are considered, and the results for $n=5,10,20$ are reported in Tables $1-3$.

(A) (Mixed portfolio) $S_{n}=X_{1}+\cdots+X_{n}$, where $X_{i} \sim \operatorname{Pareto}(2+0.1 i), i=1, \ldots, 5 ; X_{i} \sim$ $\operatorname{Exp}(i-5), i=6, \ldots, 10 ; X_{i} \sim \log -\operatorname{Normal}\left(0,(0.1(i-10))^{2}\right), i=11, \ldots, 20$.

(B) (Light-tailed portfolio) $S_{n}=Y_{1}+\cdots+Y_{n}$, where $Y_{i} \sim \operatorname{Exp}(i), i=1, \ldots, 5 ; Y_{i} \sim \operatorname{Weibull}(i-$ $5,1 / 2), i=6, \ldots, 10 ; Y_{i} \stackrel{d}{=} Y_{i-10}, i=11, \ldots, 20$.

(C) (Pareto portfolio) $S_{n}=Z_{1}+\cdots+Z_{n}$, where $Z_{i} \sim \operatorname{Pareto}(1.5), i=1, \ldots, 20$.

From Tables 1-3, we have the following observations.

(i) The worst-case VaR at level 0.975 and the worst-case ES at level 0.975 are very close, even for small numbers of $n$, in all models considered (Theorem 3.3, (3.10)).

(ii) The ratio between the worst-case VaR at level 0.975 and the worst-case ES at level 0.975 goes to 1 as $n$ grows large. In the heavy-tailed model $(\mathrm{C})$, the convergence is relatively slow (Theorem 3.3, (3.11)). 
Table 1: Bounds obtained with RA $\left(\Delta x=10^{-6}\right)$, Model (A): mixed portfolio.

\begin{tabular}{l||ccc|ccc|cccc}
\multicolumn{1}{l||}{} & \multicolumn{3}{c|}{$n=5$} & \multicolumn{3}{c|}{$n=10$} & \multicolumn{4}{c}{$n=20$} \\
& best & worst & spread & best & worst & spread & best & worst & spread \\
\hline $\operatorname{ES}_{0.975}\left(S_{n}\right)$ & 22.48 & 44.88 & 22.40 & 22.52 & 55.59 & 33.07 & 29.15 & 102.35 & 73.20 \\
$\operatorname{VaR}_{0.975}\left(S_{n}\right)$ & 9.79 & 41.46 & 31.67 & 10.04 & 52.67 & 42.63 & 21.44 & 100.65 & 79.21 \\
$\operatorname{VaR}_{0.9875}\left(S_{n}\right)$ & 12.06 & 56.21 & 44.16 & 12.06 & 69.03 & 56.98 & 22.12 & 126.63 & 104.51 \\
$\operatorname{VaR}_{0.99}\left(S_{n}\right)$ & 12.96 & 62.01 & 49.05 & 12.96 & 75.34 & 62.38 & 22.29 & 136.30 & 114.01 \\
\hline$\overline{\overline{E S}}_{0.975}\left(S_{n}\right)$ & & 1.08 & & & 1.06 & & & 1.02 &
\end{tabular}

Table 2: Bounds obtained with RA $\left(\Delta x=10^{-6}\right)$, Model (B): light-tailed portfolio.

\begin{tabular}{l||ccc|ccc|cccc}
\multicolumn{1}{l||}{} & \multicolumn{3}{c|}{$n=5$} & \multicolumn{3}{c|}{$n=10$} & \multicolumn{4}{c}{$n=20$} \\
& best & worst & spread & best & worst & spread & best & worst & spread \\
\hline $\operatorname{ES}_{0.975}\left(S_{n}\right)$ & 4.72 & 10.71 & 5.99 & 24.55 & 63.19 & 38.63 & 31.33 & 126.38 & 95.04 \\
$\operatorname{VaR}_{0.975}\left(S_{n}\right)$ & 3.69 & 10.57 & 6.88 & 13.61 & 61.41 & 47.81 & 13.61 & 125.73 & 112.13 \\
$\operatorname{VaR}_{0.9875}\left(S_{n}\right)$ & 4.38 & 12.15 & 7.77 & 19.20 & 78.75 & 59.55 & 19.20 & 160.75 & 141.55 \\
$\operatorname{VaR}_{0.99}\left(S_{n}\right)$ & 4.61 & 12.66 & 8.05 & 21.21 & 84.80 & 63.59 & 21.21 & 172.96 & 151.75 \\
\hline$\overline{\operatorname{ES}}_{0.975}\left(S_{n}\right)$ & & 1.01 & & & 1.03 & & & 1.01 &
\end{tabular}

Table 3: Bounds obtained with RA $\left(\Delta x=10^{-6}\right)$, Model (C): Pareto portfolio.

\begin{tabular}{l||ccc|ccc|ccc}
\multicolumn{1}{l||}{} & \multicolumn{3}{c|}{$n=5$} & \multicolumn{3}{c|}{$n=10$} & \multicolumn{3}{c}{$n=20$} \\
& best & worst & spread & best & worst & spread & best & worst & spread \\
\hline $\mathrm{ES}_{0.975}\left(S_{n}\right)$ & 103.8 & 172.6 & 68.8 & 166.2 & 345.1 & 178.9 & 266.2 & 690.3 & 424.1 \\
$\operatorname{VaR}_{0.975}\left(S_{n}\right)$ & 15.7 & 130.6 & 114.9 & 21.8 & 291.3 & 269.5 & 43.5 & 620.8 & 577.3 \\
$\operatorname{VaR}_{0.9875}\left(S_{n}\right)$ & 22.6 & 207.3 & 184.7 & 27.6 & 462.4 & 434.8 & 46.7 & 985.5 & 938.8 \\
$\operatorname{VaR}_{0.99}\left(S_{n}\right)$ & 25.5 & 240.5 & 215.0 & 30.5 & 536.5 & 506.0 & 47.5 & 1143.6 & 1096.0 \\
\hline$\overline{\mathrm{ES}}_{.975}\left(S_{n}\right)$ & & 1.32 & & & 1.19 & & & 1.11 &
\end{tabular}


(iii) The DU-spreads of $\mathrm{VaR}_{0.99}, \mathrm{VaR}_{0.985}$ and $\mathrm{VaR}_{0.975}$ are larger than those of $\mathrm{ES}_{0.975}$ in all considered models (Proposition 4.1).

(iv) In the heavy-tailed Model (C), the DU-spreads of VaR are significantly larger than those of ES (Remark 4.1).

\section{Conclusion}

In this paper, we considered the risk measures VaR and ES under dependence uncertainty. We introduced the notion of aggregation-robustness and showed that all coherent distortion risk measures, including ES, are aggregation-robust while VaR is not. We also derived bounds for the worst-case and best-case VaR in aggregation and its diversification ratio under dependence uncertainty. An asymptotic equivalence between VaR and ES for inhomogeneous portfolios under the weakest known conditions on the marginal distributions was established. It was shown that VaR generally exhibits a larger uncertainty spread compared to ES at the same or a lower confidence level. Numerical examples were provided to support our theoretical results. The main results in this paper suggest that VaR is less robust than ES with respect to dependence uncertainty in aggregation.

\section{Acknowledgement}

R. Wang acknowledges financial support from the Natural Sciences and Engineering Research Council of Canada (NSERC), and the Forschungsinstitut für Mathematik (FIM) at ETH Zurich during his visit in 2013. P. Embrechts thanks the Oxford-Man Institute for its hospitality during his visit as 2014 Oxford-Man Chair. The authors would like to thank Edgars Jakobsons (ETH Zurich) for his kind help on the numerical examples in this paper.

\section{A Proofs}

\section{A.1 A useful lemma}

Before presenting the main proofs, we first introduce a lemma that is essential to prove the main results in Sections 3 and 4 in this paper. Recall the definitions of the essential supremum and the essential infimum of rvs: for any rv $S$,

$$
\operatorname{ess-sup} S=\sup \{t: \mathbb{P}(S \leqslant t)<1\}
$$

and

$$
\operatorname{ess-inf} S=\inf \{t: \mathbb{P}(S \leqslant t)>0\} .
$$


We denote $S_{n}=X_{1}+\cdots+X_{n}$ in the following. We remind the reader that such $S_{n}$ is different from the symbolic one in the notation of $\overline{\operatorname{VaR}}_{p}\left(S_{n}\right)$. We hope this will not lead to notational confusion.

Lemma A.1. Suppose that $\left(F_{i}, i \in \mathbb{N}\right)$ is a sequence of distributions on $[0,1]$, then there exist $X_{i} \sim F_{i}, i \in \mathbb{N}$, such that for each $n \in \mathbb{N}$,

$$
\operatorname{ess-\operatorname {sup}} S_{n}-\operatorname{ess-inf} S_{n} \leqslant 1
$$

Proof. We first show that if $X$ and $Y$ are counter-monotonic and both take values in $[0,1]$, then ess-sup $(X+Y)-\operatorname{ess-inf}(X+Y) \leqslant 1$. Since $X$ and $Y$ are counter-monotonic, there exist $U \sim \mathrm{U}[0,1]$ such that $X=F^{-1}(U)$ and $Y=G^{-1}(1-U)$ where $F$ and $G$ are the distributions of $X$ and $Y$, respectively. For $u, v \in(0,1)$, one of $F^{-1}(u)-F^{-1}(v)$ and $G^{-1}(1-u)-G^{-1}(1-v)$ is non-positive. Hence,

$$
\begin{aligned}
& F^{-1}(u)+G^{-1}(1-u)-\left(F^{-1}(v)+G^{-1}(1-v)\right) \\
& =\left(F^{-1}(u)-F^{-1}(v)\right)+\left(G^{-1}(1-u)-G^{-1}(1-v)\right) \\
& \leqslant \max \left\{F^{-1}(u)-F^{-1}(v), G^{-1}(1-u)-G^{-1}(1-v)\right\} \\
& \leqslant 1 .
\end{aligned}
$$

Thus,

$\operatorname{ess-sup}(X+Y)-\operatorname{ess-inf}(X+Y)=\sup _{u \in(0,1)}\left\{F^{-1}(u)+G^{-1}(1-u)\right\}-\inf _{v \in(0,1)}\left\{F^{-1}(v)+G^{-1}(1-v)\right\} \leqslant 1$.

Let $X_{1} \sim F_{1}$. For $k \geqslant 2$, let $X_{k}$ be counter-monotonic with $S_{k-1}$. Since ess-sup $\left(X_{1}\right)-$ $\operatorname{ess-inf}\left(X_{1}\right) \leqslant 1$, by induction we get that $\operatorname{ess-\operatorname {sup}}\left(S_{k}\right)-\operatorname{ess-inf}\left(S_{k}\right)=\operatorname{ess-sup}\left(S_{k-1}+X_{k}\right)-$ $\operatorname{ess-inf}\left(S_{k-1}+X_{k}\right) \leqslant 1$ for all $k \geqslant 2$. This completes the proof.

Remark A.1. Lemma A.1 is of independent interest in the probability theory of negative dependence. Indeed, it shows that an extremely negatively dependent sequence always exists for bounded marginal distributions. The definition of and details on extremely negative dependence can be found in Wang and Wang (2014). In the latter paper, it was shown that an extremely negatively dependent sequence always exists for identical marginal $L_{1}$-distributions. Lemma A.1, as a new contribution, confirms that the same statement holds for inhomogeneous marginal distributions if we assume some uniform boundedness.

The following useful corollary is directly implied by Lemma A.1.

Corollary A.2. Suppose that $\left(F_{i}, i \in \mathbb{N}\right)$ is a sequence of distributions with bounded support, then there exist $X_{i} \sim F_{i}, i \in \mathbb{N}$, such that for each $n \in \mathbb{N}$,

$$
\left|S_{n}-\mathbb{E}\left[S_{n}\right]\right| \leqslant L_{n}
$$


where $L_{n}$ is the largest length of the support of $F_{i}, i=1, \ldots, n$, that is,

$$
L_{n}=\max \left\{\operatorname{ess}-\sup X_{i}-\operatorname{ess}-\inf X_{i}: X_{i} \sim F_{i}, i=1, \ldots, n\right\} .
$$

\section{A.2 Proof of Theorem 2.1}

Proof. Suppose $\rho$ is a distortion risk measure with distortion function $h$. Since $h$ is increasing and convex on $(0,1)$, its has left-derivative on $(0,1)$, denoted as

$$
\delta(t):=\lim _{x \rightarrow 0+} \frac{h(t)-h(t-x)}{x}, t \in(0,1) .
$$

Since $h$ may have a jump at 1 , we let $\delta(1)=\delta_{1}(h(1)-h(1-))$, where $\delta_{1}$ is the Dirac delta function at 1. It follows that $\rho(X)=\int_{0}^{1} \operatorname{VaR}_{t}(X) \delta(t) \mathrm{d} t$. Note that since $\mathfrak{S}_{n}$ is compatible with a coherent risk measure $\rho$, we have that $\mathbb{E}\left[\left|X_{i}\right|\right]<\infty, i=1, \ldots, n$. For $q \in(1 / 2,1)$, define

$$
\tilde{\rho}_{q}(X)=\frac{1}{1-h(q)} \int_{q}^{1} \operatorname{VaR}_{t}(X) \delta(t) \mathrm{d} t, \quad X \in L^{0} .
$$

We can easily check that $\tilde{\rho}_{q}$ is also a coherent distortion risk measure.

For any $S \in \mathfrak{S}_{n}\left(F_{1}, \ldots, F_{n}\right)$, write $S=X_{1}+\cdots+X_{n}$, where $X_{i} \sim F_{i}, i=1, \ldots, n$. For $q \in(1 / 2,1)$, we have that

$$
\begin{aligned}
\left|\rho(S)-\int_{1-q}^{q} \operatorname{VaR}_{t}(S) \delta(t) \mathrm{d} t\right| & =\left|\int_{0}^{1-q} \operatorname{VaR}_{t}(S) \delta(t) \mathrm{d} t+\int_{q}^{1} \operatorname{VaR}_{t}(S) \delta(t) \mathrm{d} t\right| \\
& \leqslant\left|\int_{0}^{1-q} \operatorname{VaR}_{t}(S) \delta(t) \mathrm{d} t\right|+\left|(1-h(q)) \tilde{\rho}_{q}(S)\right| \\
& \leqslant \delta(1-q) \int_{0}^{1-q}\left|\operatorname{VaR}_{t}(S)\right| \mathrm{d} t+\left|(1-h(q)) \tilde{\rho}_{q}(S)\right| .
\end{aligned}
$$

Note that

$$
\left|(1-h(q)) \tilde{\rho}_{q}(S)\right| \leqslant\left|(1-h(q)) \sum_{i=1}^{n} \tilde{\rho}_{q}\left(X_{i}\right)\right|=\left|\sum_{i=1}^{n} \int_{q}^{1} \operatorname{VaR}_{t}\left(X_{i}\right) \delta(t) \mathrm{d} t\right| .
$$

On the other hand, by the comonotonic additivity of $\operatorname{VaR}_{t}, t \in(0,1)$, we have that

$$
\begin{aligned}
\int_{0}^{1-q}\left|\operatorname{VaR}_{t}(S)\right| \mathrm{d} t & =\int_{0}^{1-q}\left|\operatorname{VaR}_{t}\left(S \mathrm{I}_{\{S \geqslant 0\}}\right)+\operatorname{VaR}_{t}\left(S \mathrm{I}_{\{S<0\}}\right)\right| \mathrm{d} t \\
& \leqslant \int_{0}^{1-q} \operatorname{VaR}_{t}\left(S \mathrm{I}_{\{S \geqslant 0\}}\right) \mathrm{d} t+\int_{0}^{1-q} \operatorname{VaR}_{1-t}\left(-S \mathrm{I}_{\{S<0\}}\right) \mathrm{d} t \\
& \leqslant \int_{0}^{1-q} \operatorname{VaR}_{t}(|S|) \mathrm{d} t+\int_{0}^{1-q} \operatorname{VaR}_{1-t}(|S|) \mathrm{d} t \\
& \leqslant 2(1-q) \operatorname{ES}_{q}(|S|) \\
& \leqslant(1-q) \sum_{i=1}^{n} \operatorname{ES}_{q}\left(\left|X_{i}\right|\right) \\
& =2 \sum_{i=1}^{n} \int_{q}^{1} \operatorname{VaR}_{t}\left(\left|X_{i}\right|\right) \mathrm{d} t
\end{aligned}
$$


Note that for $i=1, \ldots, n, \rho\left(X_{i}\right)<\infty$ implies that $\int_{q}^{1} \operatorname{VaR}_{t}\left(X_{i}\right) \delta(t) \mathrm{d} t \rightarrow 0$ as $q \rightarrow 1$, and that $\mathbb{E}\left[\left|X_{i}\right|\right]<\infty$ implies that $\int_{q}^{1} \operatorname{VaR}_{t}\left(\left|X_{i}\right|\right) \mathrm{d} t \rightarrow 0$ as $q \rightarrow 1$. As a consequence, as $q \rightarrow 1$,

$$
\eta(q):=\left|\rho(S)-\int_{1-q}^{q} \operatorname{VaR}_{t}(S) \delta(t) \mathrm{d} t\right| \rightarrow 0
$$

uniformly in $S \in \mathfrak{S}_{n}$. Therefore, for each $\varepsilon>0$, there exists $1 / 2<q<1$ such that $\eta(q)<\varepsilon / 3$. By Theorem 1 of Cont et al. (2010), the distortion risk measure

$$
\hat{\rho}_{q}(X):=\frac{1}{2 q-1} \int_{1-q}^{q} \operatorname{VaR}_{t}(X) \delta(t) \mathrm{d} t, \quad X \in L^{0}
$$

is continuous at all distributions with respect to the Lévy distance. In summary, for each $\varepsilon>0$, and $q \in(1 / 2,1)$ fixed, there exists $\delta>0$ such that $\left|\hat{\rho}_{q}(S)-\hat{\rho}_{q}(T)\right|<\varepsilon / 3$ for all $S, T$ such that $d(S, T)<\delta$. Therefore, for all $S, T \in \mathfrak{S}_{n}$ with $d(S, T)<\delta$, we have that

$$
|\rho(S)-\rho(T)| \leqslant(2 q-1)\left|\hat{\rho}_{q}(S)-\hat{\rho}_{q}(T)\right|+2 \eta(q)<\varepsilon .
$$

Thus, $\rho$ is aggregation-robust.

\section{A.3 Proof of Theorem 3.1}

Proof. We will use the following equivalence lemma in Bernard et al. (2014); in the latter paper, an alternative definition of VaR is used:

$$
\operatorname{VaR}_{p}^{*}(X)=\inf \{x \in \mathbb{R}: \mathbb{P}(X \leqslant x)>p\}, \quad p \in(0,1) .
$$

Lemma A.3 (Lemma 4.3 of Bernard et al. (2014)). For $p \in(0,1)$ and a continuous distribution $F$,

$$
\sup _{S \in \mathfrak{S}_{n}} \operatorname{VaR}_{p}^{*}(S)=\sup \left\{\operatorname{ess}-\inf S: S \in \mathfrak{S}_{n}\left(F_{p, 1}, \ldots, F_{p, n}\right)\right\}
$$

and

$$
\inf _{S \in \mathfrak{S}_{n}} \operatorname{VaR}_{p}(S)=\inf \left\{\operatorname{ess}-\sup S: S \in \mathfrak{S}_{n}\left(F_{1}^{p}, \ldots, F_{n}^{p}\right)\right\}
$$

where $F_{p, i}$ is the conditional distribution of $W_{i} \sim F_{i}$ on $\left[F_{i}^{-1}(p), \infty\right)$, and $F_{i}^{p}$ is the conditional distribution of $W_{i} \sim F_{i}$ on $\left(-\infty, F_{i}^{-1}(p)\right), i=1, \ldots, n$.

For the proof of Theorem 3.1, we first show that for $p \in(0,1)$ and $q \in(p, 1]$,

$$
\sup \left\{\operatorname{ess}-\inf S: S \in \mathfrak{S}_{n}\left(F_{p, 1}, \ldots, F_{p, n}\right)\right\} \geqslant \sum_{i=1}^{n} \mu_{p, q}^{(i)}-\max _{i=1, \ldots, n}\left(F_{i}^{-1}(q)-F_{i}^{-1}(p)\right) .
$$

Since the case when $F_{i}^{-1}(q)=\infty$ for some $i$ is trivial, we suppose that $F_{i}^{-1}(q)<\infty$ for all $i=1, \ldots, n$. 
Let $F_{p, q}^{(i)}$ be the conditional distribution of $W_{i} \sim F_{i}$ on $\left[F_{i}^{-1}(p), F_{i}^{-1}(q)\right]$ for $0<p<q \leqslant 1$. By Corollary A.2, there exist rvs $X_{i} \sim F_{p, q}^{(i)}, i=1, \ldots, n$, such that

$$
X_{1}+\cdots+X_{n} \geqslant \sum_{i=1}^{n} \mu_{p, q}^{(i)}-\max _{i=1, \ldots, n}\left(F^{-1}(q)-F^{-1}(p)\right) .
$$

Let $Z_{i}, i=1, \ldots, n$ be any $\mathrm{rv}$ with distribution $F_{q, i}$, and let $C$ be a set independent of $X_{1}, \ldots, X_{n}, Z_{1}, \ldots, Z_{n}$ and with $\mathbb{P}(C)=(q-p) /(1-p)$. Define $Y_{i}=X_{i} \mathrm{I}_{C}+Z_{i}\left(1-\mathrm{I}_{C}\right)$ for $i=1, \ldots, n$. It is straightforward to check that $Y_{i}$ has distribution $F_{p, i}$, and

$$
Y_{1}+\cdots+Y_{n} \geqslant X_{1}+\cdots+X_{n} \geqslant \sum_{i=1}^{n} \mu_{p, q}^{(i)}-\max _{i=1, \ldots, n}\left(F_{i}^{-1}(q)-F_{i}^{-1}(p)\right) .
$$

Thus

$$
\operatorname{ess}-\inf \left(Y_{1}+\cdots+Y_{n}\right) \geqslant \sum_{i=1}^{n} \mu_{p, q}^{(i)}-\max _{i=1, \ldots, n}\left(F_{i}^{-1}(q)-F_{i}^{-1}(p)\right),
$$

and we obtain (A.3). Since $\operatorname{VaR}_{p}(X) \geqslant \operatorname{VaR}_{r}^{*}(X)$ for any $r<p$ and any $\operatorname{rv} X$, we have that

$$
\begin{aligned}
\overline{\operatorname{VaR}}_{p}\left(S_{n}\right) \geqslant \lim _{r \rightarrow p-}\left(\sup _{S \in \mathfrak{S}_{n}} \operatorname{VaR}_{p}^{*}(S)\right) & \geqslant \lim _{r \rightarrow p-}\left(\sum_{i=1}^{n} \mu_{r, q}^{(i)}-\max _{i=1, \ldots, n}\left(F_{i}^{-1}(q)-F_{i}^{-1}(r)\right)\right) \\
& =\sum_{i=1}^{n} \mu_{p, q}^{(i)}-\max _{i=1, \ldots, n}\left(F_{i}^{-1}(q)-F_{i}^{-1}(p)\right) .
\end{aligned}
$$

Note that here we use the fact that $F_{i}^{-1}$ is left-continuous for each $i$. On the other hand,

$$
\overline{\operatorname{VaR}}_{p}\left(S_{n}\right) \leqslant \sup _{S \in \mathfrak{S}_{n}} \operatorname{VaR}_{p}^{*}(S)=\sup \left\{\operatorname{ess}-\inf S: S \in \mathfrak{S}_{n}\left(F_{p}, \ldots, F_{p}\right)\right\} \leqslant \sum_{i=1}^{n} \mu_{p, 1}^{(i)}
$$

always holds. Thus we obtain (3.5). We can show (3.6) similarly.

\section{A.4 Proof of Theorem 3.3}

Proof. First, let us assume that $\mathbb{E}\left[X_{i}\right]=0$ for all $i \in \mathbb{N}$. Note that $\overline{\operatorname{ES}}_{p}\left(S_{n}\right)=\sum_{i=1}^{n} \operatorname{ES}_{p}\left(X_{i}\right)=$ $\sum_{i=1}^{n} \mu_{p, 1}^{(i)}$ for $X_{i} \sim F_{i}$. We use (3.5) and take $q_{n}=1-n^{-1}$ for $n$ large enough such that $q_{n}>p$. By (b), we have $\sum_{i=1}^{n} \mu_{p, 1}^{(i)}>0$ for large $n$.

Note that by (a), $\mathbb{E}\left[\left|X_{i}\right|^{k}\right] \leqslant M$ uniformly. Therefore, $\left[F_{i}^{-1}(t)\right]^{k}(1-t) \leqslant M$ for $t \in(0,1)$, and we have

$$
F_{i}^{-1}(t) \leqslant\left(\frac{M}{1-t}\right)^{1 / k}, \quad t \in(0,1), i \in \mathbb{N}
$$


Note that for $X_{i} \sim F_{i}$,

$$
\begin{aligned}
\mu_{p, 1}^{(i)}-\mu_{p, q_{n}}^{(i)} & =\frac{1}{1-p} \mathbb{E}\left[X_{i} \mathrm{I}_{\left\{X_{i} \geqslant F_{i}^{-1}(p)\right\}}\right]-\frac{1}{q_{n}-p} \mathbb{E}\left[X_{i} \mathrm{I}_{\left\{F_{i}^{-1}\left(q_{n}\right) \geqslant X_{i} \geqslant F_{i}^{-1}(p)\right\}}\right] \\
& \leqslant \frac{1}{1-p} \mathbb{E}\left[X_{i} \mathrm{I}_{\left\{X_{i} \geqslant F^{-1}\left(q_{n}\right)\right\}}\right] \\
& =\frac{1}{1-p} \int_{q_{n}}^{1} F_{i}^{-1}(t) \mathrm{d} t \\
& \leqslant \frac{1}{1-p} \int_{q_{n}}^{1}\left(\frac{M}{1-t}\right)^{1 / k} \mathrm{~d} t \\
& =\frac{1}{1-p} \frac{1}{1-1 / k} M^{1 / k}\left(1-q_{n}\right)^{1-1 / k}
\end{aligned}
$$

As a consequence we have

$$
\begin{aligned}
\sup _{S \in \mathfrak{S}_{n}} \operatorname{VaR}_{p}(S) & \geqslant \sum_{i=1}^{n} \mu_{p, q_{n}}^{(i)}-\max _{i=1, \ldots, n}\left(F_{i}^{-1}\left(q_{n}\right)-F_{i}^{-1}(p)\right) \\
& \geqslant \sum_{i=1}^{n} \mu_{p, 1}^{(i)}-\sum_{i=1}^{n}\left(\mu_{p, 1}^{(i)}-\mu_{p, q_{n}}^{(i)}\right)-\max _{i=1, \ldots, n} F_{i}^{-1}\left(q_{n}\right) \\
& \geqslant \sum_{i=1}^{n} \mu_{p, 1}^{(i)}-\sum_{i=1}^{n} \frac{1}{1-p} \frac{1}{1-1 / k} M^{1 / k}\left(1-q_{n}\right)^{1-1 / k}-\left(\frac{M}{1-q_{n}}\right)^{1 / k} \\
& =\sum_{i=1}^{n} \mu_{p, 1}^{(i)}-\frac{1}{1-p} \frac{1}{1-1 / k} M^{1 / k} n^{1 / k}-M^{1 / k} n^{1 / k} \\
& =\sum_{i=1}^{n} \mu_{p, 1}^{(i)}-O\left(n^{1 / k}\right) .
\end{aligned}
$$

By (b), it follows that

$$
1 \geqslant \frac{\overline{\operatorname{VaR}}_{p}\left(S_{n}\right)}{\sum_{i=1}^{n} \mu_{p, 1}^{(i)}} \geqslant 1-\frac{O\left(n^{1 / k}\right)}{\sum_{i=1}^{n} \mu_{p, 1}^{(i)}} \rightarrow 1 \text { as } n \rightarrow \infty,
$$

hence we obtain (3.10).

Now for the case that $\mathbb{E}\left[X_{i}\right] \neq 0$ for some $i \in \mathbb{N}$, we denote by $F_{i}^{*}$ the distribution of $X_{i}-\mathbb{E}\left[X_{i}\right]$, and by

$$
\mathfrak{S}_{n}^{*}=\left\{Y_{1}+\cdots+Y_{n}: Y_{i} \sim F_{i}^{*}, i=1, \ldots, n\right\} .
$$

Then, by (A.4), with $\mathfrak{S}_{n}$ replaced by $\mathfrak{S}_{n}^{*}$, we have

$$
\begin{aligned}
\sup _{S \in \mathfrak{S}_{n}} \operatorname{VaR}_{p}(S) & =\sup _{S \in \mathfrak{S}_{n}^{*}} \operatorname{VaR}_{p}(S)+\sum_{i=1}^{n} \mathbb{E}\left[X_{i}\right] \\
& =\sum_{i=1}^{n}\left(\mu_{p, 1}^{(i)}-\mathbb{E}\left[X_{i}\right]\right)-O\left(n^{1 / k}\right)+\sum_{i=1}^{n} \mathbb{E}\left[X_{i}\right] \\
& =\sum_{i=1}^{n} \mu_{p, 1}^{(i)}-O\left(n^{1 / k}\right) .
\end{aligned}
$$

Thus, (A.4) still holds for $\mathfrak{S}_{n}$ in the case $\mathbb{E}\left[X_{i}\right] \neq 0$ for some $i$. 
When (b*) holds, by (A.4), we have that

$$
1 \geqslant \frac{\overline{\operatorname{VaR}}_{p}\left(S_{n}\right)}{\sum_{i=1}^{n} \mu_{p, 1}^{(i)}} \geqslant 1-\frac{\left(\frac{1}{1-p} \frac{k}{k-1}+1\right) M^{1 / k}\left(n^{1 / k}\right)}{\sum_{i=1}^{n} \mu_{p, 1}^{(i)}} \geqslant 1-C n^{-1+1 / k},
$$

for $n$ sufficiently large. This leads to (3.11) and completes the proof of the theorem.

\section{A.5 Proof of Corollary 4.1}

Proof. (i) By Theorem 3.3 and Corollary 3.4, we have that

$$
\lim _{n \rightarrow \infty} \frac{\overline{\operatorname{VaR}}_{p}\left(S_{n}\right)-\underline{\operatorname{VaR}}_{p}\left(S_{n}\right)}{\overline{\operatorname{ES}}_{p}\left(S_{n}\right)-\underline{\mathrm{LES}}_{p}\left(S_{n}\right)}=1,
$$

and (4.1) follows directly, noting that $\operatorname{ES}_{p}(X) \geqslant \mathbb{E}[X] \geqslant \operatorname{LES}_{p}(X)$ for any $\operatorname{rv} X$ and any $p \in(0,1)$.

(ii) Corollary 3.3 of Wang and Wang (2014) implies that

$$
\lim _{n \rightarrow \infty} \frac{\underline{E S}_{p}\left(S_{n}\right)}{n}=\mathbb{E}[X],
$$

and hence we obtain (4.2).

\section{References}

Acerbi, C. (2002). Spectral measures of risk: A coherent representation of subjective risk aversion. Journal of Banking and Finance, 26(7), 1505-1518.

Artzner, P., Delbaen, F., Eber, J.-M. and Heath, D. (1999). Coherent measures of risk. Mathematical Finance, 9(3), 203-228.

BCBS (2012). Consultative Document May 2012. Fundamental review of the trading book. Basel Committee on Banking Supervision. Basel: Bank for International Settlements.

BCBS (2013). Consultative Document October 2013. Fundamental review of the trading book: A revised market risk framework. Basel Committee on Banking Supervision. Basel: Bank for International Settlements.

Ben-Tal, A., El Ghaoui, L. and Nemirovski, A. (2009). Robust optimization. Princeton University Press, New Jersey.

Bernard, C., Jiang, X. and Wang, R. (2014). Risk aggregation with dependence uncertainty. Insurance: Mathematics and Economics, 54, 93-108. 
Cheung, K. C., and Lo, A. (2013). General lower bounds on convex functionals of aggregate sums. Insurance: Mathematics and Economics, 53(3), 884-896.

Cont, R., Deguest, R. and Scandolo, G. (2010). Robustness and sensitivity analysis of risk measurement procedures. Quantitative Finance 10(6), 593-606.

Daníelsson, J., Jorgensen, B. N., Mandira, S., Samorodnitsky, G. and de Vries, C. G. (2005). Subadditivity re-examined: the case for Value-at-Risk. Discussion paper, 549. Financial Markets Group, London School of Economics and Political Science.

Dobrushin, R. (1970). Prescribing a system of random variables by conditional distributions. Theory of Probability and its Applications, 15(3), 458-486.

Embrechts, P. and Puccetti, G. (2006). Bounds for functions of dependent risks. Finance and Stochastics, 10, 341-352.

Embrechts, P., Puccetti, G. and Rüschendorf, L. (2013). Model uncertainty and VaR aggregation. Journal of Banking and Finance, 37(8), 2750-2764.

Embrechts, P., Puccetti, G., Rüschendorf, L., Wang, R. and Beleraj, A. (2014). An academic response to Basel 3.5. Preprint, ETH Zurich.

Emmer, S., Kratz, M. and Tasche, D. (2014). What is the best risk measure in practice? A comparison of standard measures. Preprint, ESSEC Business School.

Föllmer, H. and Schied, A. (2011). Stochastic finance: an introduction in discrete time. Third ed., Walter de Gruyter, Berlin/New York.

EIOPA (2011). Equivalence assessment of the Swiss supervisory system in relation to articles 172, 227 and 260 of the Solvency II Directive, EIOPA-BoS-11-028, available at https:// eiopa . europa. eu/consultations/consultation-papers/index.html.

Gneiting, T. (2011). Making and evaluating point forecasts. Journal of the American Statistical Association 106(494), 746-762.

Hampel, F. R., Ronchetti, E. M., Rousseeuw, P. J. and Stahel, W. A. (1986). Robust statistics: the approach based on influence functions. Wiley, New York.

Hansen, L. P. and Sargent, T. J. (2007). Robustness. Princeton University Press, New Jersey.

Huber, P. J. and Ronchetti E. M. (2009). Robust statistics. Second ed., Wiley Series in Probability and Statistics. Wiley, New Jersey. 
Jorion, P. (2006). Value at Risk: the new benchmark for managing financial risk. Third ed., McGraw-Hill, New York.

Kou, S. and Peng, X. (2014). On the measurement of economic tail risk. Preprint, Hong Kong University of Science and Technology.

Krätschmer, V., Schied, A. and Zähle, H. (2014). Comparative and quantitiative robustness for law-invariant risk measures. Finance and Stochastics, to appear.

Kusuoka, S. (2001). On law invariant coherent risk measures. Advances in Mathematical Economics, 3, 83-95.

Makarov, G. D. (1981). Estimates for the distribution function of the sum of two random variables with given marginal distributions. Theory of Probability and its Applications 26, 803-806.

Nelsen, R. (2006). An introduction to copulas. Second ed., Springer, New York.

Puccetti, G. (2013). Sharp bounds on the expected shortfall for a sum of dependent random variables. Statistics and Probability Letters, 83 (4), 1227-1232

Puccetti, G. and Rüschendorf, L. (2013). Sharp bounds for sums of dependent risks. Journal of Applied Probability, 50(1), 42-53.

Puccetti, G. and Rüschendorf, L. (2014). Asymptotic equivalence of conservative value-at-riskand expected shortfall-based capital charges. Journal of Risk, 16(3), 1-19.

Puccetti, G., Wang, B. and Wang, R. (2013). Complete mixability and asymptotic equivalence of worst-possible VaR and ES estimates. Insurance: Mathematics and Economics, 53(3), 821828.

Rüschendorf, L. (1982). Random variables with maximum sums. Advances in Applied Probability, 14(3), 623-632.

Sandström, A. (2010). Handbook of solvency for actuaries and risk managers: theory and practice. Taylor \& Francis US, Florida.

SCOR (2008). From principle-based risk management to solvency requirements. Second ed., SCOR, Zurich. SCOR Switzerland AG.

Wang, B. and Wang, R. (2011). The complete mixability and convex minimization problems for monotone marginal distributions. Journal of Multivariate Analysis, 102, 1344-1360.

Wang, B. and Wang, R. (2014). Extreme negative dependence and risk aggregation. Preprint, University of Waterloo. 
Wang, R. (2014). Asymptotic bounds for the distribution of the sum of dependent random variables. Journal of Applied Probability, to appear.

Wang, R., Peng, L. and Yang, J. (2013). Bounds for the sum of dependent risks and worst Value-at-Risk with monotone marginal densities. Finance and Stochastics, 17(2), 395-417.

Wang, S. S., Young, V. R. and Panjer, H. H. (1997). Axiomatic characterization of insurance prices. Insurance: Mathematics and Economics, 21(2), 173-183. 